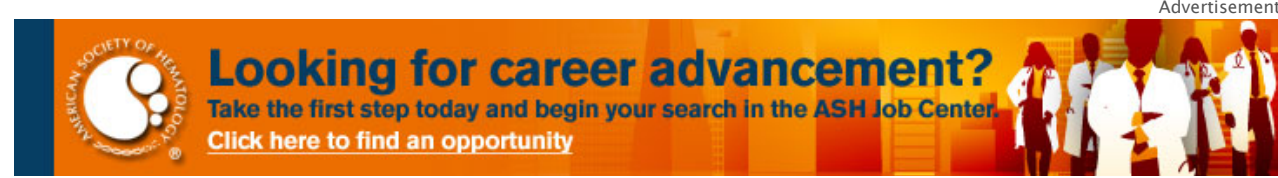

ASH Home Blood App My Folders Alerts RSS

$$
\text { search }
$$

Advanced Search

| Home $\mid$ About Blood $\mid$ Aut

Authors

\begin{tabular}{l|l|l} 
Submit to Blood & Subscriptions & Classifieds
\end{tabular}

All Issues

Home / December 3, 2015; Blood: 126 (23)

\title{
The Sea Urchin sns 5 Chromatin Insulator Improves the Likelihood of Lentiviral Vectors in Erythroid Milieu By Organizing an Independent Chromatin Domain at the Integration Site
}

Elena Baiamonte*, ${ }^{1}$, Rosalia Di Stefano*, ${ }^{1}$, Melania Lo Iacono*, ${ }^{1}$, Barbara Spina*, ${ }^{\text {, }}$, Angela Vitrano*, ${ }^{1}$, Rosario Di Maggio* ${ }^{1}$, Massimiliano Sacco*, , Giovanni Spinelli*,2, Aurelio Maggio', Santina Acuto*, ${ }^{1}$, and Vincenzo Cavalieri*,2,3

Author Affiliations

1 Campus of Haematology Franco e Piera Cutino- A.O.O.R. "Villa Sofia-Cervello", Palermo, Italy

2 Department of Biological, Chemical and Pharmaceutical Sciences and Technologies (STEBICEF), University of Palermo, Palermo, Italy

3 Mediterranean Center for Human Health Advanced Biotechnologies (CHAB), University of Palermo, Palermo, Italy

\section{Abstract}

Retroviral vectors are currently the most suitable vehicles for therapeutic gene transfer in hematopoietic stem cells. However, these vectors are known to integrate rather randomly throughout the genome, suffering the so called chromosomal position effects (PE). Such a critical occurrence most probably depends upon the ability of heterochromatin to spread in the inserted vector sequences. Moreover, the use of transgenes imply genotoxicity effects, since the cis-regulatory sequences harbored by the vector can disturb the proper transcription of the resident genes neighboring the integration site, potentially leading to malignant transformation.

Due to their enhancer blocker activity, the incorporation of chromatin insulators in flanking position to the transferred unit can reduce the mentioned dangerous effects. Moreover, by acting as barriers to the spread of heterochromatin, chromatin insulators can also mitigate vector silencing.

We have previously shown that the sea urchin sns 5 chromatin insulator activity is conserved in mouse and human erythroid milieu: it blocks the Bglobin-LCR-HS2 enhancer/globin promoter interaction when placed between them. In addition, when placed in flanking location of a $y$-retrovirus vector, sns5 impedes PE variegation and improves vector-specific expression following integration in the erythroid genome. Importantly, by binding both erythroid-specific and ubiquitous factors, sns 5 favors the accumulation inside the provirus locus of epigenetic marks commonly associated to an euchromatic state (Acuto S. et al., BCMD 2005; D'Apolito D. et al., 2009; Di Caro D. et al., J Mol Biol 2004; Cavalieri V. et al., NAR 2009).

In this study we extend these findings, demonstrating that sns 5 works as

Volume: 126
Issue: 23
Pages: $4414-4414$
DOI: http://dx.doi.org/
$\checkmark$ Citation Alert

Article

Info \& Metrics

E-Letters

Related Articles

No related articles found.

Cited By... 
2/2/2016 Blood Journal | The Sea Urchin sns5 Chromatin Insulator Improves the Likelihood of Lentiviral Vectors in Erythroid Milieu By Organizing an Independ...

chromatin insulator also when placed in flanking position of a GFP transgene

contained in a lentivirus vector (LV-GFP). A large panel of mouse

erythroleukemic clones (MELC) was generated after transduction with

uninsulated and sns 5 -insulated LV-GFP. Individual clones were screened for

single vector integrants (by Q-PCR), and for GFP-expression (by

cytofluorimetry). Our results shown that the inclusion of the sns 5 element in

a forward orientation increased the fraction of vector expressing cells ( $89 \%$

for the insulated vector vs $42 \%$ for the uninsulated ones). The clonal

variegation of expression, assessed as frequency of clones that showed a

percentage of GFP-negative cells in the progeny, decreased in clones

transduced with the insulated vectors ( $7.4 \%$ vs $13,9 \%)$.

It has been suggested that chromatin insulators could shape the architecture of topologically independent chromosome domains. High resolution mapping of chromosomal domains in drosophila and higher eukaryotes highlighted that chromatin insulators play a critical role in shaping the architectural genome organization both in a local chromosome environment and in long range chromosomal interaction. Intriguingly, by using the Chromosome Conformation Capture (3C) technology, we demonstrated that the sns 5 flanked LV-GFP integrated at a single copy in the erythroid cell genome is organized into an independent chromatin loop at the integration site. Worth to mention, no looping was detected in the absence of sns5, indicating that the two flanking copies of sns 5 are specifically involved in the reorganization of the chromatin structure at the provirus locus.

In conclusion our results not only confirm the conserved and striking boundary function of sns5, but also provide a new clue concerning the molecular mechanism that allows this function to occur. On these basis, our findings reassure the use of sns 5 to improve both efficacy and safety of lentiviral vectors for gene therapy.

This work was funded by the Assessorato Regionale della Salute, Regione Siciliana (PO FESR 4.1.1.1 RIMEDRI)

Disclosures No relevant conflicts of interest to declare.

- $\downarrow^{*}$ Asterisk with author names denotes non-ASH members

Articles by Baiamonte, E.

Articles by Cavalieri, V.

Articles by Baiamonte, E.

Articles by Cavalieri, V.

C 2015 by The American Society of Hematology

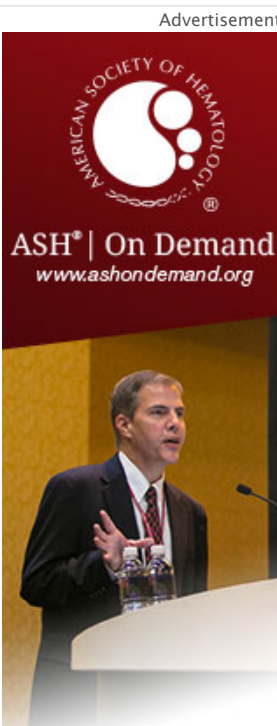

How will you treat your patients with the latest available agents?

LEARN FROM THE EXPERTS

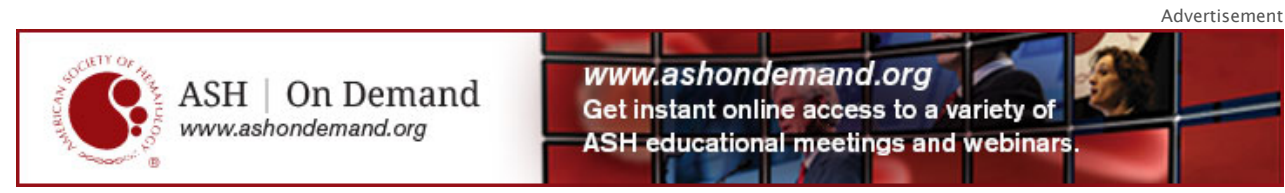

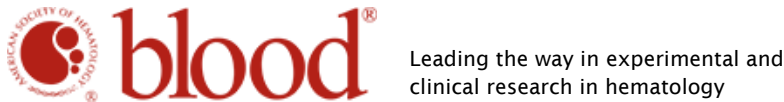

American Society of Hematology 2021 L Street NW, Suite 900, Washington, DC 20036 Phone 202-776-0544 | Fax 202-776-0545

\author{
Current Issue \\ First Edition \\ Collections \\ All Issues
}

Abstracts

Video Library
Subscriptions

About Blood

Newsroom

Public Access

Permissions

Order Reprints
Submit to Blood

Alerts

RSS

Blood App

Contact Us

Feedback
Information for:

Authors

Subscribers

Institutions/Librarians

Advertising in Blood

ASH Privacy Policy 
